\title{
SUCCESSFUL THERAPY OF METASTATIC BREAST CANCER WITH LIFE-THREATENING BONE MARROW FAILURE DURING THE COVID-19 PANDEMIC: REFLECTIONS ON A CASE REPORT
}

Jorge Illarramendi', Richard Breeze1, Montserrat Outeiro', Idoia Mugica', Esteban Salgado², Jose Juan Illarramendi²

'Service of Hematology, Complejo Hospitalario de Navarra - Pamplona, Spain.

${ }^{2}$ Service of Medical Oncology, Complejo Hospitalario de Navarra - Pamplona, Spain.

Introduction: Delayed diagnoses and treatments of breast cancer $(\mathrm{BC})$ have been described as a consequence of the COVID19 crisis. Some cases may even progress to a situation of functional derangements or so-called visceral crises before the start of the treatment. We described a case of $\mathrm{BC}$ with life-threatening pancytopenia who needed urgent therapy during the peak of the first wave of the COVID-19 pandemic. Case Report: A 52-year-old lady was diagnosed with Her2/neupositive BC with bone metastases in March 2020. During the waiting time for a positron emission tomography scan, she attended the emergency room in a very bad condition, with extreme asthenia, disorientation, and dyspnea. Altered blood analyses include severe anemia (hemoglobin $6 \mathrm{~g} / \mathrm{dL}$ ) and thrombocytopenia (4,000 platelets/ $\mu \mathrm{L}$ ) with a leukoerythroblastic reaction. Bone marrow biopsy confirmed invasion by BC. Polymerase chain reaction for SARS-CoV-2 was negative. Our hospital was collapsed at that time, with more than two-thirds of beds occupied by COVID-19 patients. On Easter Saturday (April 11), we started urgent chemotherapy with weekly reduced doses of paclitaxel $\left(30 \mathrm{mg} / \mathrm{m}^{2}\right)$ combined with trastuzumab (TTZ). Multiple transfusions of red cells and platelets were also needed. Standard doses were implemented after an initial improvement, and pertuzumab (PTZ) was also added in combination with TTZ. After 5 months, a complete response (CR) was documented by imaging and normalization of tumor markers. Therapy has been continued with PTZ + TTZ every 3 weeks. In March 2021, she maintains a status of CR and enjoys a normal active life. Conclusions: Situations of hospital crises like COVID-19 require individualized and adjusted protocols and schedules to deal with abnormal situations not fulfilling classical criteria used in clinical trials. Chemotherapy and target therapy may be successful in some of these patients. The use of available supportive care measures is also the main factor for optimal results.

Keywords: Breast Cancer; Bone Marrow Invasion; COVID-19; Paclitaxel; Pertuzumab; Trastuzumab. 